\title{
Can reactogenicity predict immunogenicity after COVID-19 vaccination?
}

\author{
Young Hoon Hwang ${ }^{1,}$, , Kyoung-Ho Song ${ }^{2,}$, Yunsang Choi ${ }^{2}$, Suryeong Go ${ }^{2}$, Su-Jin Choi ${ }^{3}$, Jongtak Jung ${ }^{2}$, \\ Chang Kyung Kang ${ }^{1}$, Pyoeng Gyun Choe ${ }^{1}$, Nam-Joong Kim ${ }^{1}$, Wan Beom Park ${ }^{1}$, and Myoung-don Oh${ }^{1}$
}

\begin{abstract}
${ }^{1}$ Department of Internal Medicine, Seoul National University Hospital, Seoul; ${ }^{2}$ Department of Internal Medicine, Seoul National University Bundang Hospital, Seongnam; ${ }^{3}$ Laboratory of Infection \& Immunity, Seoul National University Hospital Biomedical Research Institute, Seoul, Korea
\end{abstract}

Received: April 29, 2021

Revised : May 11, 2021

Accepted: May 11, 2021

\section{Correspondence to}

Wan Beom Park, M.D.

Department of Internal

Medicine, Seoul National

University Hospital, 101 Daehak-

ro, Jongno-gu, Seoul 03080,

Korea

Tel: +82-2-2072-3596

Fax: $+82-2-762-9662$

E-mail: wbpark1@snu.ac.kr

https://orcid.org/0000-0003-

0022-9625

*These authors contributed equally to this work
Background/Aims: This study aimed to assess the association between local and systemic reactogenicity and humoral immunogenicity after severe acute respiratory syndrome coronavirus 2 (SARS-CoV-2) vaccination.

Methods: Adverse events were prospectively evaluated using an electronic diary in 135 healthy adults who received a SARS-CoV-2 vaccine (AZD1222, AstraZeneca/ Oxford, $\mathrm{n}=42$; or BNT162b2, Pfizer/BioNTech, $\mathrm{n}=93$ ). We semi-quantitatively measured anti-S1 immunoglobulin G (IgG) using an enzyme-linked immunosorbent assay at baseline, 3 weeks after the first dose of AZD1222 or BNT162b2, and 2 weeks after the second dose of BNT162b2. We evaluated the association between the maximum grade of local or systemic adverse events and the anti-S1 IgG optical density using multivariate linear regression with adjustment for age, sex, and use of antipyretics.

Results: The median age of the 135 vaccinees was 30 years ( 36 years in the AZD1222 group and 29 years in the BNT162b2 group) and $25.9 \%$ were male (9.5\% in the AZD1222 group and $33.3 \%$ in the BNT162b2 group). Local and systemic adverse events were generally comparable after the first dose of AZD1222 and the second dose of BNT162b2. The grades of local and systemic adverse events were not significantly associated with anti-S1 IgG levels in the AZD1222 or BNT162b2 group. Conclusions: Local and systemic reactogenicity may not be associated with humoral immunogenicity after SARS-CoV-2 vaccination.

Keywords: COVID-19; SARS-CoV-2; Immunogenicity, vaccine; Drug-related side effects and adverse reactions; Enzyme-linked immunosorbent assay

\section{INTRODUCTION}

The coronavirus disease 2019 (COVID-19) global epidemic continues despite the introduction of vaccines against severe acute respiratory syndrome coronavirus 2 (SARS-CoV-2) [1]. In South Korea, the government began a nationwide COVID-19 vaccination program in February 2021. Two types of vaccines are currently available in South Korea, namely, a chimpanzee adenovirus-vectored vaccine called AZD1222 (AstraZeneca/Oxford) [2,3] and a mRNA-based vaccine called BNT162b2 (Pfizer/BioNTech) [4].

Safety profiles from major clinical trials showed that mild-to-moderate adverse reactions to the vaccines are common, and a prospective survey in South Korea yielded similar results [5]. However, the clinical implication of vaccine reactogenicity is uncertain. In addition, although the efficacy of COVID-19 vaccines has been proven by randomized clinical trials [2-4], vaccinees may be concerned about antibody formation at an individu- 
al level. Some experts argue that vaccine reactogenicity correlates with immunogenicity, while others insist this is not always the case $[6,7]$.

The correlation between reactogenicity and immunogenicity after vaccination has been poorly studied, especially for novel SARS-CoV-2 vaccines. Here, we aimed to evaluate the association between reactogenicity and immunogenicity for two SARS-CoV-2 vaccines by performing a prospective survey of adverse events and measuring humoral immunity.

\section{METHODS}

\section{Study population and design}

This study included healthy healthcare workers who planned to receive a SARS-CoV-2 vaccine in Seoul National University Hospital (SNUH) and Seoul National University Bundang Hospital (SNUBH) between March and April 2021. Although participants in the AZD1222 group were scheduled to receive two injections 10 weeks apart, they did not receive the second injection during the study period. Participants in the BNT162b2 group received two injections 3 weeks apart.

Serum samples were collected at baseline, 3 weeks after the first injection of AZD1222 or BNT162b2, and 2 weeks after the second injection of BNT162 b2 and stored at $-70^{\circ} \mathrm{C}$. Participants reported solicited and unsolicited adverse events from the day of vaccination (day o) to 7 days after vaccination (day 7) using an electronic diary-based self-report form. This study was approved by the Institutional Review Board of SNUH (2102-0321193) and SNUBH (B-2102-669-302). All participants provided written informed consent.

\section{Adverse event assessment}

Local (injection site pain, swelling, and redness) and systemic (vomiting, diarrhea, headache, fatigue, chills, muscle pain, joint pain, and fever) adverse events were assessed using the U.S. Food and Drug Administration guidance [8]. Participants reported use of antipyretics during the self-report period. Grade o was no adverse events in all categories. Adverse events were graded as follows: Grade 1, did not interfere with activity; Grade 2, interfered with activity; Grade 3, prevented daily activity; and Grade 4, required an emergency department visit or hospitalization. Exceptions were as follows: local redness and swelling (Grade 1, 2.5 to $5.0 \mathrm{~cm}$ in diameter; Grade 2, 5.0 to $10.0 \mathrm{~cm}$ in diameter; Grade 3, > $10 \mathrm{~cm}$ in diameter; and Grade 4, necrosis or exfoliative dermatitis), vomiting (Grade 1, 1 to 2 times in 24 hours; Grade 2, > 2 times in 24 hours; Grade 3, required intravenous hydration; and Grade 4, required an emergency department visit or hospitalization due to hypovolemic shock), diarrhea (Grade 1, 2 to 3 loose stools in 24 hours; Grade 2, 4 to 5 loose stools in 24 hours; Grade 3, six or more loose stools in 24 hours; and Grade 4, required an emergency department visit or hospitalization), and fever (Grade 1, $38.0^{\circ} \mathrm{C}$ to $38.4^{\circ} \mathrm{C}$; Grade $2,38.5^{\circ} \mathrm{C}$ to $38.9^{\circ} \mathrm{C}$; Grade $3,39.0^{\circ} \mathrm{C}$ to $40.0^{\circ} \mathrm{C}$; and Grade $4,>40^{\circ} \mathrm{C}$ ).

\section{Measurement of anti-S1 immunoglobulin G by a semi-quantitative enzyme-linked immunosorbent assay}

Antibody immunoglobulin G (IgG) against the spike protein subunit $(\mathrm{S} 1)$ of SARS-CoV-2 was semi-quantitatively measured using an enzyme-linked immunosorbent assay (ELISA) kit (EUROIMMUN, Mountain Lakes, NJ, USA) [9]. The optical density (O.D.) ratio was interpreted as follows: $<0.8$, negative; $\geq 0.8$ to $<1.1$, borderline; and $\geq 1.1$, positive. Borderline was considered negative for analysis.

\section{Statistical analysis}

The association between the highest grade of local or systemic adverse events and the anti-S1 IgG O.D. ratio in post-vaccination serum was evaluated using a multivariate linear regression model. The anti-S IgG O.D. ratio was set as the dependent variable and the highest grade of local or systemic adverse events was set as the independent variable. Additional covariates were age, sex, and use of antipyretics. $p$ values less than 0.05 were considered statistically significant. All statistical analyses were conducted using IBM SPSS Statistics version 26.o for Windows (IBM, Armonk, NY, USA).

\section{RESULTS}

\section{Study population}

A total of 135 healthy healthcare workers who received a SARS-CoV-2 vaccine were enrolled in this study. Among 

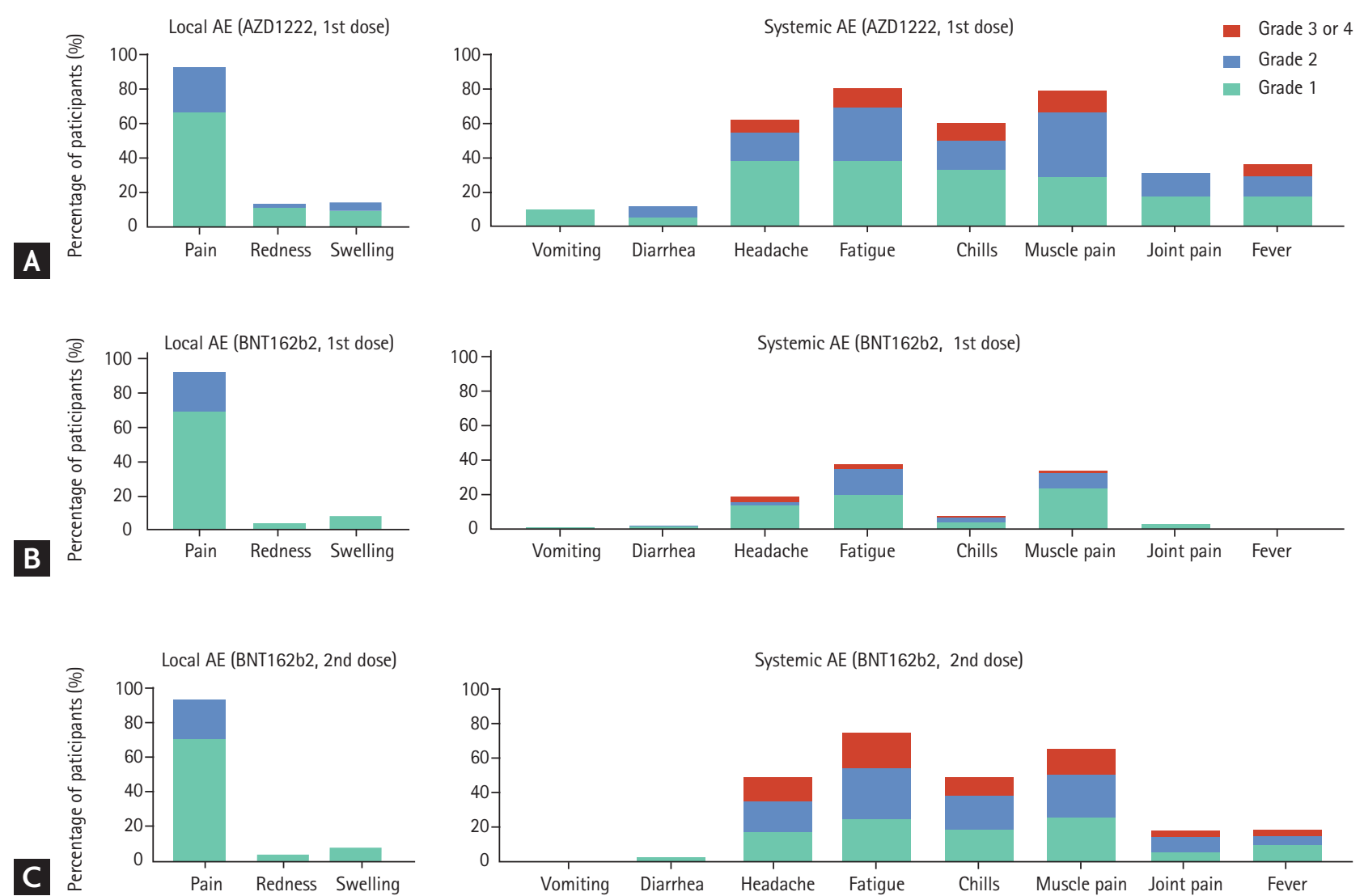

Figure 1. Local and systemic adverse events (AEs) reported within 7 days after AZD1222 (AstraZeneca) or BNT162b2 (Pfizer) vaccination. Local (left) and systemic (right) AEs were assessed for 7 days after each vaccination using an electronic diary form. Solicited AEs are shown after the first injection of AZD1222 (A) and after the first (B) and second (C) injections of BNT162b2.

them, 42 participants received AZD1222 and 93 participants received $\mathrm{BNT}_{1} 62 \mathrm{~b} 2$. Their median age was 30 years 36 years in the AZD1222 group and 29 years in the BNT162b2 group) and $25.9 \%$ were male (9.5\% in the AZD1222 group and $33.3 \%$ in the $\mathrm{BNT}_{1} 62 \mathrm{~b} 2$ group).

\section{Local and systemic reactogenicity after SARS-CoV-2 vaccination}

Among the 42 participants who received the first injection of AZD1222, 39 (93\%) reported a local adverse event and $40(95 \%)$ reported a systemic adverse event. The most common adverse event was local pain (93\%), followed by fatigue ( $81 \%)$, muscle pain (79\%), and headache (62\%) (Fig. 1A). Eleven (26\%) participants reported moderate or severe local adverse events (Grade $\geq 2$ ), 30 (71\%) reported moderate or severe systemic adverse events, and $36(86 \%)$ took antipyretics. One person visited the emergency department due to severe fatigue and weak- ness 15 minutes after receiving AZD1222. Her symptoms improved after 3 hours of close observation and she was discharged.

In the BNT162b2 group, adverse events were more common and severe after the second injection than after the first injection (Fig. $1 \mathrm{~B}$ and $1 \mathrm{C}$ ). Among the 93 participants who received the second injection of BNT162 b2, 85 (91\%) reported a local adverse event, of which 37 (40\%) were moderate-to-severe. Seventy-six $(82 \%)$ reported a systemic adverse event, of which 56 (60\%) were moderate-to-severe, and 68 (73\%) took antipyretics. Local and systemic adverse events were comparable after the first injection of AZD1222 and the second injection of BNT162b2. However, fever was more common in the AZD1222 group than in the BNT162b2 group (36\% vs. $19 \%, p=0.042$ ). 


\section{Immunogenicity after vaccination}

One of the 42 participants in the AZD1222 group showed serologic positivity at baseline (O.D. ratio 1.38) and was excluded from the analysis. The baseline anti-S1 IgG O.D. ratio in the AZD1222 group was 0.24 (95\% confidence interval [CI], 0.18 to 0.29$)$. Serologic positivity was observed in $88 \%$ ( 36 of 41 ) of participants at 3 weeks after the first injection of AZD1222, and their mean anti-S1 IgG O.D. ratio was 4.76 (95\% CI, 3.84 to 5.67 ) (Fig. $2 \mathrm{~A}$ and $2 \mathrm{~B}$ ).

In the BNT162b2 group, serum samples were collected from 40 participants after the first injection and from 93 participants after the second injection. Among them, one participant was excluded from the analysis due to serologic positivity at baseline (O.D. ratio 1.25). The baseline anti-S1 IgG O.D ratio was 0.15 (95\% CI, o.11 to 0.19). Serologic positivity was observed in $100 \%$ of participants at 3 weeks after the first injection of BNT162 b2 (39 of 39), and their mean anti-S1 IgG O.D. ratio was 10.37 (95\% CI, 9.51 to 11.23) (Fig. $2 \mathrm{C}$ and $2 \mathrm{D}$ ). After the second injection, all 92 BNT162b2 recipients showed serologic positivity, and their mean anti-S1 IgG O.D. ratio was 10.56 (95\% CI, 10.16 to 10.97 ) (Fig. $2 \mathrm{E}$ and $2 \mathrm{~F}$ ).

\section{Association of reactogenicity and humoral immuno- genicity}

A multivariate linear regression model showed that the grades of local $(p=0.129)$ and systemic $(p=0.324)$ adverse events after the first injection of AZD1222 were not significantly associated with the anti-S1 IgG O.D. ratio after adjustment for age, sex, and use of antipyretics (Supplementary Table 1). Similarly, the grades of local and systemic adverse events after the first and second injections of BNT162b2 were not significantly associated with the anti-S1 IgG O.D. ratio. Age was significantly associated with the anti-S1 IgG level after the first injection of BNT162b2 $(p=0.006)$, and age and female sex were significantly associated with the anti-S1 IgG level after the second injection of $\mathrm{BNT}_{1} 62 \mathrm{~b}_{2}$ ( $p=0.036$ and $p=0.009$, respectively).

\section{DISCUSSION}

To the best of our knowledge, this is the first study to evaluate the association between reactogenicity and humoral immunogenicity for novel SARS-CoV-2 vaccines.
This study showed that the severity of local and systemic adverse events was not associated with anti-SARS-CoV2-specific IgG levels after vaccination with AZD1222 or BNT162b2. These findings may be helpful for counseling vaccinees and managing vaccine adverse events.

Reactogenicity refers to a subset of reactions that occur after vaccination and are commonly manifested with inflammation [10]. In a clinical setting, reactogenicity after vaccination is linked to safety and is widely surveyed by the severity and frequency of adverse events. However, it is unclear whether a more reactogenic vaccine elicits a more immunogenic response. Some studies of vaccine adjuvants suggest that there is a correlation between reactogenicity and humoral and cellular immune responses; however, the results are inconsistent $[6,7,10]$.

In the case of SARS-CoV-2 vaccination, previous studies reported that $\mathrm{BNT} 162 \mathrm{~b} 2$ is less reactogenic and immunogenic in an older population consistent with the current study, but the humoral response after two doses exceeds that in convalescent serum $[4,11]$. However, the direct correlation between adverse events and immunogenicity has not been evaluated. Our study explored the association between reactogenicity and the humoral response after SARS-CoV-2 vaccination following adjustment for age, sex, and use of antipyretics. We showed that reactogenicity to AZD1222 and BNT162b2 was not associated with humoral immunogenicity. Therefore, the severity of adverse events cannot be used as a surrogate marker of vaccine efficacy. Reactogenicity is influenced by the immunologic properties of vaccines and many other factors such as physicochemical properties, administration-related factors, and intrinsic host factors, and therefore cannot be assumed to correlate with immunogenicity [10].

This study has several limitations. First, the severity of adverse events was assessed based on a self-report, which could be subjective. Second, only the maximum severity of adverse events was analyzed and the duration of adverse events was not considered. Third, only two vaccines were evaluated, and other SARS-CoV-2 vaccines may yield different results. Finally, humoral immunity was assessed by an anti-S1 IgG semi-quantitative ELISA rather than by a neutralizing antibody assay. However, the results obtained using this ELISA kit correlate well with live virus neutralizing antibody titers [12].

In conclusion, anti-SARS-CoV-2-specific antibody 


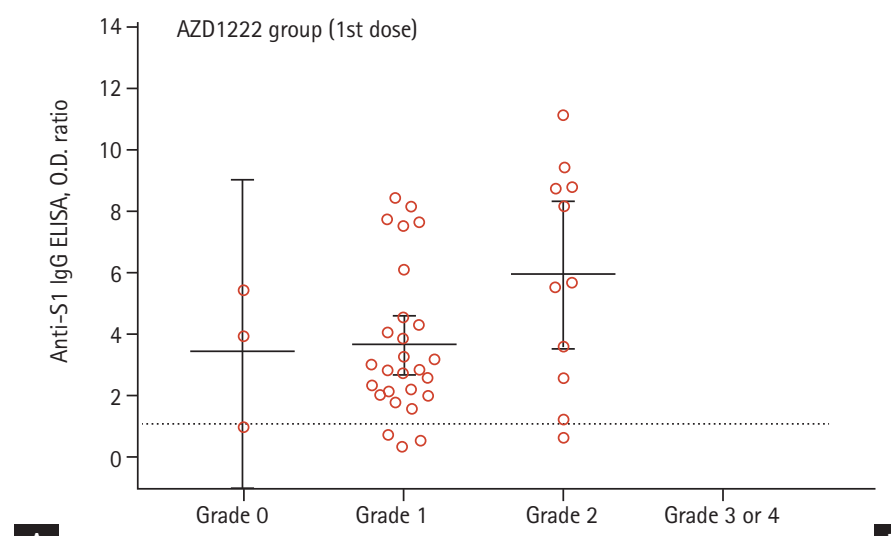

A Any local adverse event

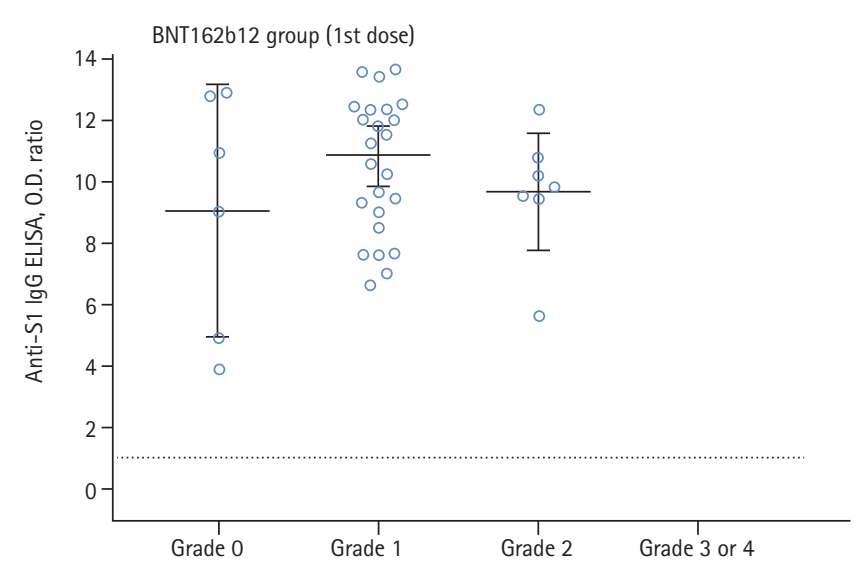

C

Any local adverse event

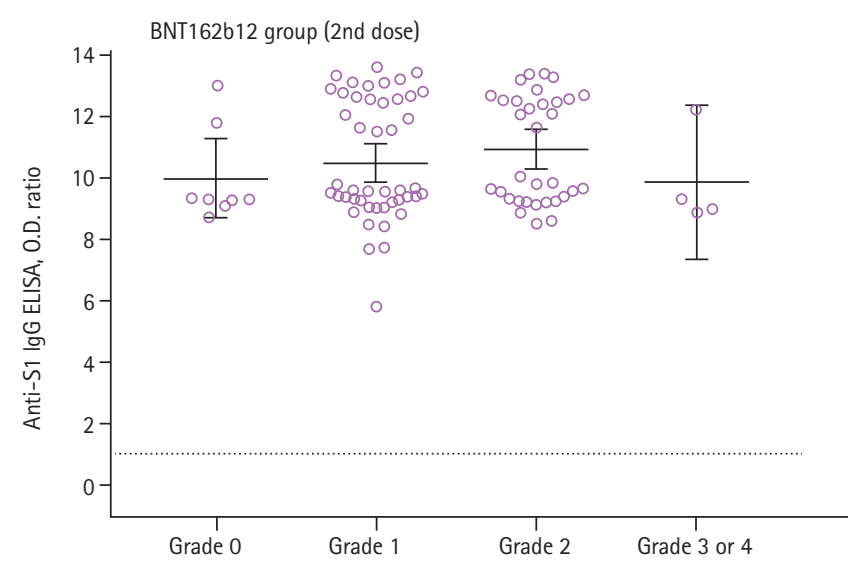

E

Any local adverse event

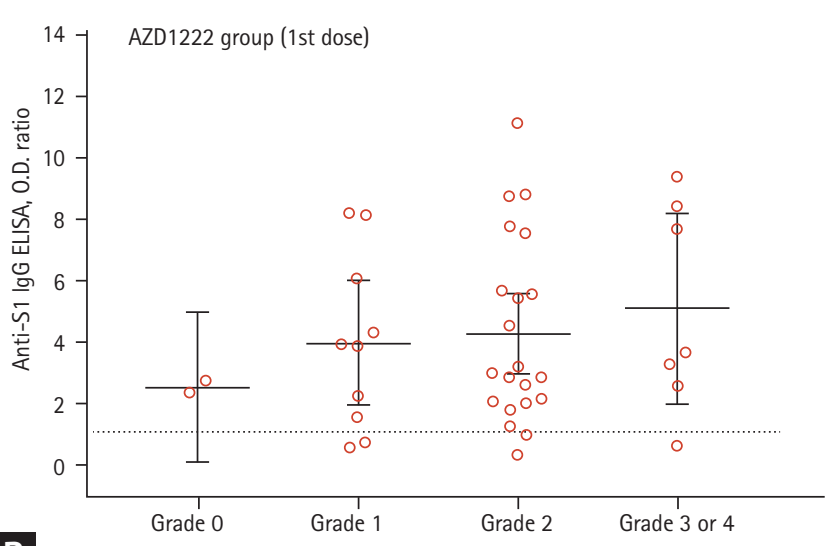

B

Any systemic adverse event

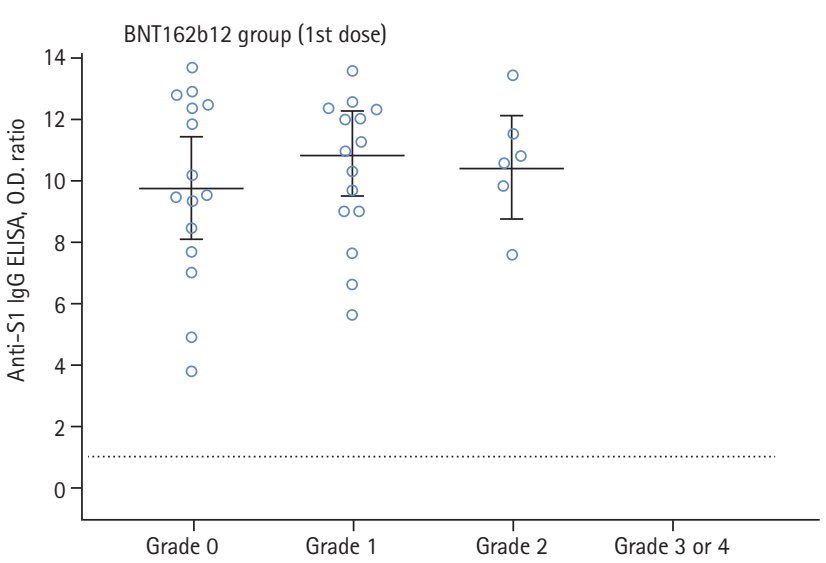

D

Any systemic adverse event

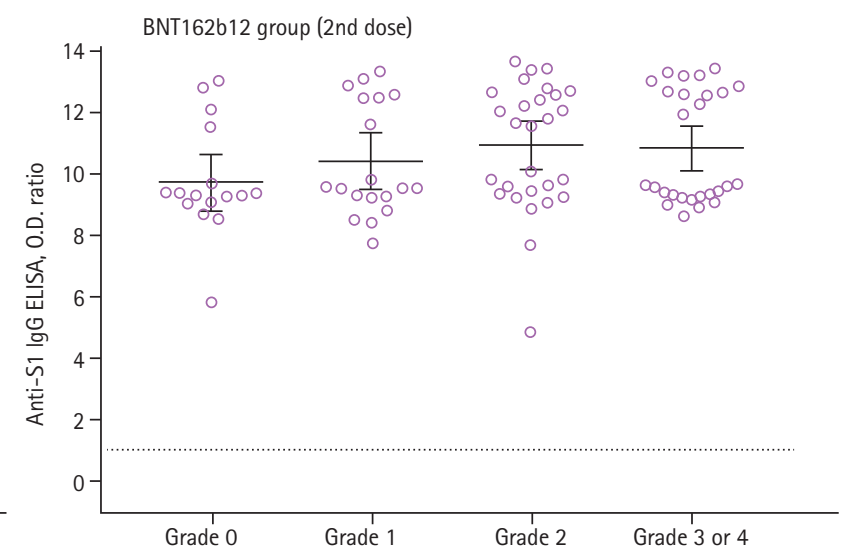

$\mathbf{F}$

Any systemic adverse event

Figure 2. Anti-S1 immunoglobulin G (IgG) enzyme-linked immunosorbent assay (ELISA) results according to the severity of local and systemic adverse events. Anti-S1 IgG ELISA optical density (O.D.) ratios were plotted according to the maximum grade of any local and systemic adverse event after the first injection of AZDi222 (A, B) and after the first (C, D) and second (E, F) injections of BNT162b2. Dotted lines denote the cutoff value of a positive anti-S1 IgG O.D. ratio (O.D. ratio $\geq 1.1$ ). Vertical and horizontal lines denote the mean and 95\% confidence interval of the anti-S1 IgG O.D. ratio, respectively. 
levels are not associated with the severity of local or systemic adverse events following vaccination with AZD1222 or BNT162b2. Our findings suggest that humoral immunogenicity cannot be predicted by reactogenicity after SARS-CoV-2 vaccination.

\section{KEY MESSAGE}

1. Local and systemic adverse events were generally comparable after the first dose of AZD1222 and the second dose of BNT162b2.

2. Local and systemic reactogenicity cannot predict humoral immunogenicity after AZD1222 or BNT162b2 vaccination.

\section{Conflict of interest}

No potential conflict of interest relevant to this article was reported.

\section{Acknowledgments}

This work was supported by grant No. 04-2021-0010 from the SNUH Research Fund and grant No. 14-2021023 from the SNUBH Research Fund.

\section{REFERENCES}

1. World Health Organization. WHO coronavirus (COVID-19) dashboard [Internet]. Geneva (CH): WHO, c2021 [cited 2021 April 26]. Available from: https://covid19.who.int/.

2. Ramasamy MN, Minassian AM, Ewer KJ, et al. Safety and immunogenicity of ChAdOxı $\mathrm{nCoV}-19$ vaccine administered in a prime-boost regimen in young and old adults (COVoo2): a single-blind, randomised, controlled, phase 2/3 trial. Lancet 2021;396:1979-1993.
3. Voysey M, Clemens SA, Madhi SA, et al. Safety and efficacy of the ChAdOxı nCoV-19 vaccine (AZD1222) against SARS-CoV-2: an interim analysis of four randomised controlled trials in Brazil, South Africa, and the UK. Lancet 2021;397:99-111.

4. Polack FP, Thomas SJ, Kitchin N, et al. Safety and efficacy of the BNT162b2 mRNA Covid-19 vaccine. N Engl J Med 2020;383:2603-2615.

5. Bae S, Lee YW, Lim SY, et al. Adverse reactions following the first dose of ChAdOxı $n \mathrm{CoV}-19$ vaccine and BNT162b2 vaccine for healthcare workers in South Korea. J Korean Med Sci 2021;36:e115.

6. Burny W, Callegaro A, Bechtold V, et al. Different adjuvants induce common innate pathways that are associated with enhanced adaptive responses against a model antigen in humans. Front Immunol 2017;8:943.

7. Mitchell TC, Casella CR. No pain no gain?: adjuvant effects of alum and monophosphoryl lipid A in pertussis and HPV vaccines. Curr Opin Immunol 2017;47:17-25.

8. Food and Drug Administration. Guidance for industry: toxicity grading scale for healthy adult and adolescent volunteers enrolled in preventive vaccine clinical trials [Internet]. Silver Spring (MD): FDA, 2007 [cited 2021 Apr 26]. Available from: https://www.fda.gov/media/73679/ download.

9. Choe PG, Kang CK, Suh HJ, et al. Waning antibody responses in asymptomatic and symptomatic SARS-CoV-2 infection. Emerg Infect Dis 2021;27:327-329.

10. Herve C, Laupeze B, Del Giudice G, Didierlaurent AM, Tavares Da Silva F. The how's and what's of vaccine reactogenicity. NPJ Vaccines 2019;4:39.

11. Walsh EE, Frenck RW Jr, Falsey AR, et al. Safety and immunogenicity of two RNA-based Covid-19 vaccine candidates. N Engl J Med 2020;383:2439-2450.

12. Choe PG, Kang CK, Suh HJ, et al. Antibody responses to SARS-CoV-2 at 8 weeks postinfection in asymptomatic patients. Emerg Infect Dis 2020;26:2484-2487. 


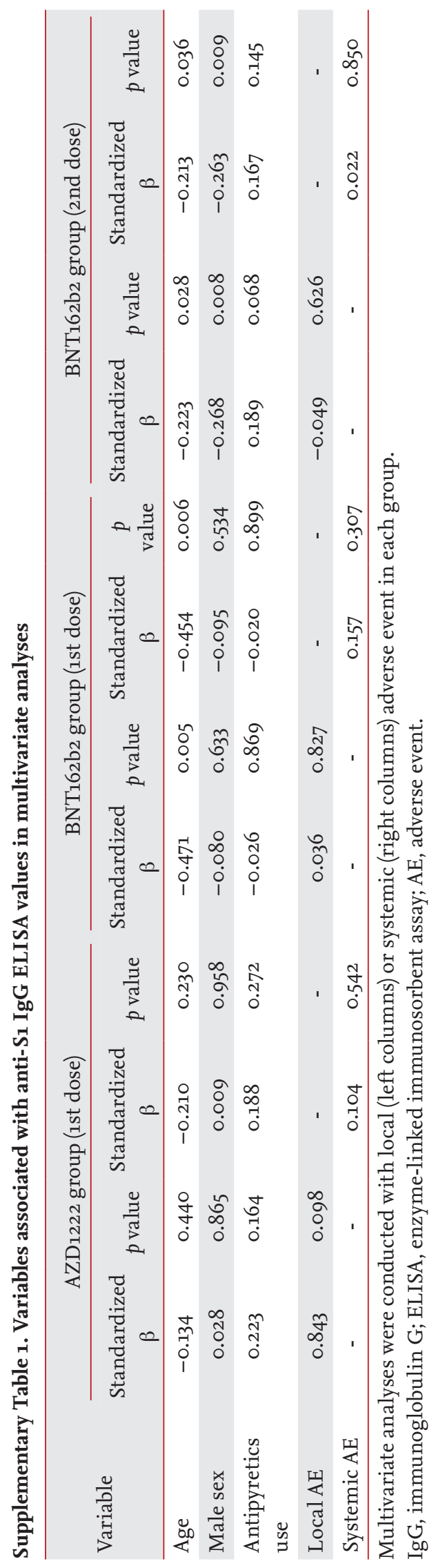

